\title{
Two new species of Tanaopsis (Tanaidacea: Tanaopsidae) from Admiralty Bay (Antarctica), with an identification key
}

\author{
Juliana L. Segadilha* and Catarina L. Araújo-Silva
}

(JLS) Laboratório de Carcinologia, Museu Nacional, Universidade Federal do Rio de Janeiro. Quinta da Boa Vista, s/n, 20940-040 Rio de Janeiro, Brazil. E-mail: julianasegadilha@gmail.com. * Corresponding author (CLAS) AEE (Laboratório de Ecologia Aquática e Evolução), CIIMAR (Centro Interdisciplinar de Investigação Marinha e Ambiental), Universidade do Porto, Rua dos Bragas, 289, 4050-123 Porto, Portugal; Fundação Capes, Ministério da Educação do Brasil, 70040-020 Brasília, DF, Brazil. E-mail: katelas@hotmail.com

\begin{abstract}
Two new species of family Tanaopsidae, genus Tanaopsis, from Admiralty Bay (Antarctica) are herein described. Tanaopsis brevicorpus sp. nov. displays antennule article 1 about 1.5 times as long as wide, carapace as long as first three pereonites, both rami of uropod biarticulate with fusion line, and pleotelson short, more than twice longer than wide. Tanaopsis bamberi sp. nov. resembles other Antarctic species T. kerguelenensis in its habitus, antennule long and uropod uniarticulate. However, the new species can be distinguished from it by a combination of characters that includes maxilliped basis with long seta arising from tubercles; inner margin of cheliped fixed finger with three smooth sub-terminal spines; uropod subequal to pleotelson in length, and uropodal endopod about twice as long as exopod.
\end{abstract}

Key words: Crustacea, Peracarida, Tanaidomorpha, Southern Ocean, King George Island.

\section{INTRODUCTION}

The monotypic family Tanaopsidae BłażewiczPaszkowycz and Bamber, 2012 is characterized mainly by the pleon being laterally convex and wider than pereon, and the cheliped fixed finger having a bifid terminal spine. The systematics of the genus Tanaopsis Sars, 1899 is confused. The genus was first described in the family Tanaidae Dana, 1849, then transferred to Paratanaidae Lang, 1949 by Lang (1949) and later relocated to Leptognathiidae Sieg, 1976 by Sieg (1976). The Paratanaoidea phylogeny performed by Larsen and Wilson (2002), and then revised by Bird and Larsen (2009), placed Tanaopsis as genus incertae sedis mainly due to the cheliped fixed finger with bifid terminal spine and coxa of pereopod-1 with a developed spur, which do not allow its inclusion in other family (Błażewicz-Paszkowycz and Bamber, 2012). Currently the genus includes 14 described species (Anderson, 2013) and it is distributed through shallow waters to bathyal and even abyssal depths, usually in muddy-sand or mud (Bird and Bamber, 2000).
Błażewicz-Paszkowycz and Bamber (2012) pointed if Tanaopsis itself is monophyletic, stating that appears to be two groups of species: one with a pointed mandibular molar process and biarticulate uropod rami [Tanaopsis antarctica Lang, 1967; T. cadieni Sieg and Dojiri, 1991; T. canaipa Bamber, 2008; T. curtus Kudinova-Pasternak, 1984; T. gallardoi (Shiino, 1970); T. oios Błażewicz-Paszkowycz and Bamber, 2012 and T. profunda Lang, 1967] and the other without a mandibular molar process and with uniarticulate uropod rami ( $T$. boonwurrungi Błażewicz-Paszkowycz and Bamber, 2012; T. chotkarakde Bird and Bamber, 2000; T. kerguelenensis Shiino, 1978 and T. rawhitia Bird, 2011). Tanaopsis laticaudatus (Sars, 1882) would fit in the first group as it has two segmented uropod rami, although it is described as being without a mandibular molar (Sars, 1899; Błażewicz-Paszkowycz and Bamber, 2012).

This work is based on Antarctic species of Tanaopsis and, herein, we describe two new species (T. bamberi sp. nov. and T. brevicorpus sp. nov.) and, additionally, an identification key to all species of the genus, based on female characters is given. 


\section{Material ANd Methods}

Specimens were collected from about 50-500 $m$ depth in Admiralty Bay (South Shetland Islands, Antarctic), during the austral summer of 2005 and 2010 (Tab. 1). The sediment was collected using a box corer, washed in a mesh size of $0.5 \mathrm{~mm}$, fixed in $4 \%$ borax buffered formalin and subsequently preserved in $70 \%$ alcohol.

Table 1. Study area and their sites and geographic coordinates containing Tanaopsis bamberi sp. nov. and Tanaopsis brevicorpus sp. nov. BP: Botany Point.

\begin{tabular}{lccccc}
\hline $\begin{array}{l}\text { Depth } \\
(\mathrm{m})\end{array}$ & Site & $\begin{array}{c}\text { Replicate } \\
\text { samples }\end{array}$ & $\begin{array}{c}\text { Sampling } \\
\text { date }\end{array}$ & $\begin{array}{c}\text { Latitude } \\
(\mathrm{S})\end{array}$ & $\begin{array}{c}\text { Longitude } \\
(\mathrm{W})\end{array}$ \\
\hline 55.7 & BP \#1 & $\mathrm{R} 1$ & $29 / \mathrm{Jan} / 05$ & $62^{\circ} 05^{\prime} 44.3^{\prime \prime}$ & $58^{\circ} 20^{\prime} 57.6^{\prime \prime}$ \\
58.2 & BP \#1 & R2 & $03 / \mathrm{Feb} / 05$ & $62^{\circ} 05^{\prime} 44.1^{\prime \prime}$ & $58^{\circ} 20^{\prime} 58.1^{\prime \prime}$ \\
58.6 & BP \#2 & R1 & $09 / \mathrm{Feb} / 05$ & $62^{\circ} 05^{\prime} 47.6^{\prime \prime}$ & $58^{\circ} 20^{\prime} 31.0^{\prime \prime}$ \\
58.6 & BP \#2 & R2 & $05 / \mathrm{Dec} / 04$ & $62^{\circ} 05^{\prime} 47.3^{\prime \prime}$ & $58^{\circ} 20^{\prime} 31.4^{\prime \prime}$ \\
64.3 & BP & R3 & $26 / \mathrm{Jan} / 04$ & $62^{\circ} 05^{\prime} 42.1^{\prime \prime}$ & $58^{\circ} 20^{\prime} 44.5^{\prime \prime}$ \\
120 & $\# 1$ & - & $07 / \mathrm{Dec} / 09$ & $62^{\circ} 05^{\prime} 54.0^{\prime \prime}$ & $58^{\circ} 25^{\prime} 46.9^{\prime \prime}$ \\
125 & $\# 2$ & - & $07 / \mathrm{Dec} / 09$ & $62^{\circ} 06^{\prime} 20.5^{\prime \prime}$ & $58^{\circ} 26^{\prime} 46.6^{\prime \prime}$ \\
117 & $\# 3$ & - & $10 / \mathrm{Dec} / 09$ & $62^{\circ} 05^{\prime} 39.6^{\prime \prime}$ & $58^{\circ} 21^{\prime} 13.8^{\prime \prime}$ \\
500.8 & $\# 1$ & - & $02 / \mathrm{Dec} / 09$ & $62^{\circ} 09^{\prime} 52.4^{\prime \prime}$ & $58^{\circ} 23^{\prime} 43.5^{\prime \prime}$ \\
400 & $\# 3$ & - & $02 / \mathrm{Dec} / 09$ & $62^{\circ} 11^{\prime} 24.8^{\prime \prime}$ & $58^{\circ} 19^{\prime} 43.0^{\prime \prime}$ \\
\hline
\end{tabular}

Dissections were made in glycerin using chemically-sharpened tungsten wire needles. Body length was measured from the tip of the cephalothorax to the apex of the pleotelson. Terminology follows Larsen (2003). Three specimens from each new species collected in Admiralty Bay were dissected and habitus and appendage drawings were made from one dissected specimen from each one, excepted the labium of T. brevicorpus sp. nov., which was recovered from another specimen dissected.

The type materials and the other specimens are deposited at the collection in the National Museum, Universidade Federal do Rio de Janeiro, Brazil (MNRJ).

\section{Results AND Discussion}

Family Tanaopsidae Błażewicz-Paszkowycz and Bamber, 2012

\section{Genus Tanaopsis Sars, 1899}

\section{Tanaopsis bamberi sp. nov.}

(Figs. 1-2)
Material examined. Holotype. One adult non-ovigerous female, length $4.6 \mathrm{~mm}$ (MNRJ 25509); station Botany Point $1 \mathrm{R} 1 ; 62^{\circ} 05^{\prime} 44.3$ "S 58²0'57.6”W; $55.7 \mathrm{~m}$; 29 January 2005. Paratype. One adult non-ovigerous female, length $4.5 \mathrm{~mm}$, dissected (MNRJ 23402); same locality as holotype. Two adult non-ovigerous females (MNRJ 23402); station Botany Point; same locality as holotype. One adult nonovigerous female (MNRJ 24441); station \#3, 6205’39.6”S 58²1'13.8”W; $117 \mathrm{~m}, 10$ December 2009. One adult non-ovigerous female, length $4.1 \mathrm{~mm}$, dissected (MNRJ 24442); \#3, 62¹1'24.8”S 58¹9'43.0”W; 400 m; 2 December 2009.

Diagnosis: Female. Antennule elongated, first and second articles 2.7 and 1.6 times as long as wide, respectively. Antenna with six articles, second and third articles with simple setae, seta on second article longer than on third article. Inner distal margin of cheliped fixed finger with three smooth spines. Uropodal exopod and endopod uniarticulate and endopod long with 2.5 times as long as exopod.

Etymology: The name is dedicated to the late Dr. Roger Bamber in recognition of his great contribution to tanaidacean knowledge.

Description: Female paratype, $4.5 \mathrm{~mm}$ (dissected).

Body (Fig. 1A) about six times as long as wide. Cephalothorax subtriangular, 1.1 times as wide as long, shorter than first three pereonites combined; with one lateral simple medial seta in each side; rostrum rounded. Pereonites. All pereonites wider than long, with slightly rounded corners. Pereonite 1 shortest, about 0.32 as long as carapace. Pereonite 3 longer than pereonites 2 and 6 and shorter than pereonite 5 . Pereonite 4 longest. Pleonite 5 longest. Pleotelson longer than two last pleonites combined (about 35\% length of pleon), with two pairs of simple and one pair of plumose terminal setae; apex pointed.

Antennule (Fig. 1B) about as long as cephalothorax. Article 1 narrow, about 2.75 times as long as wide, inner proximal margin with one simple seta, one distal inner simple seta and four plumose and one outer simple seta. Article 2 about 


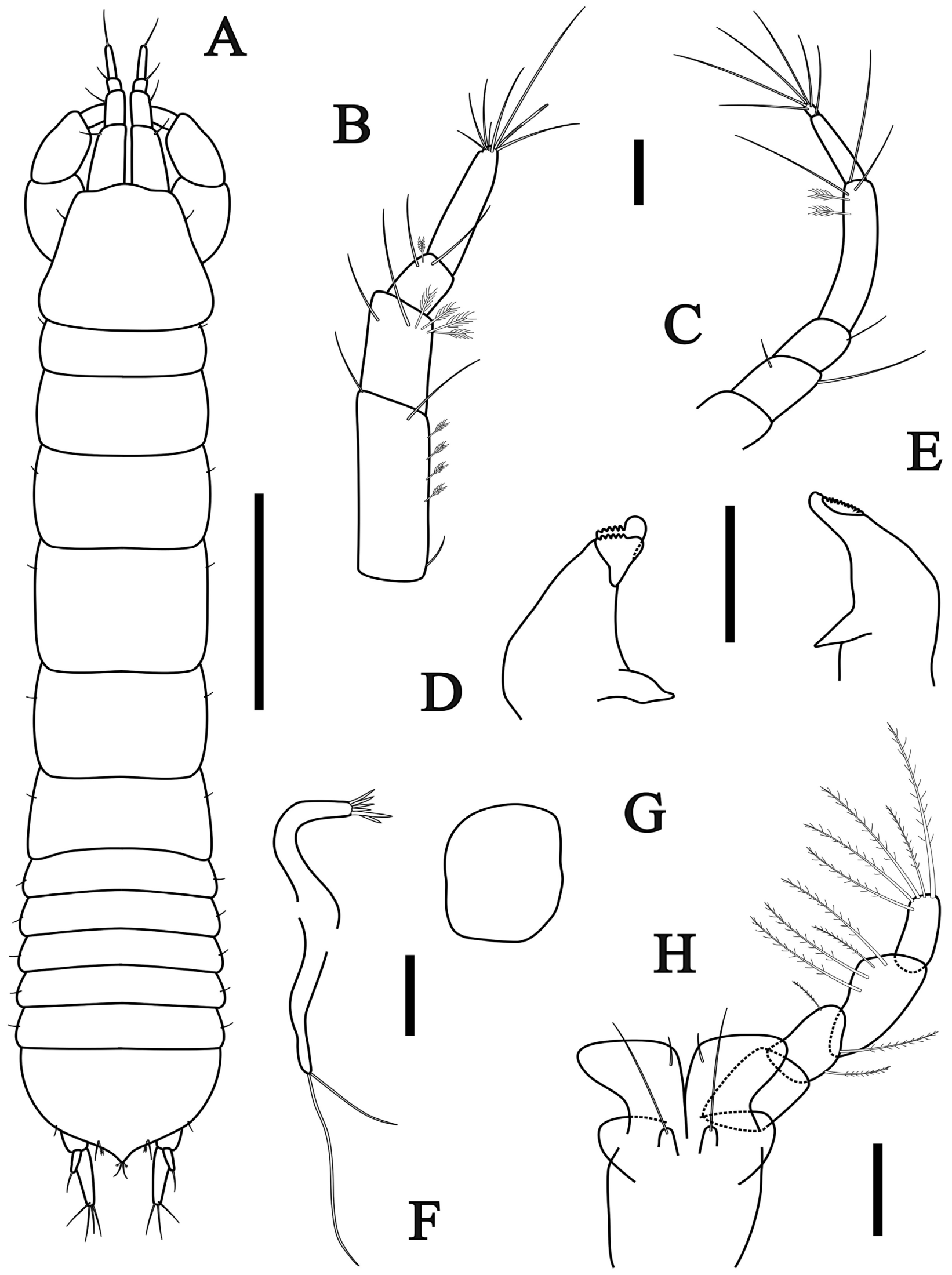

Figure 1. Tanaopsis bamberi sp. nov., female paratype, length $4.5 \mathrm{~mm}$, MNRJ 23402. A, dorsal view; B, antennule; C, antenna; D, left mandible; E, right mandible; F, maxillule; $G$, maxilla; $H$, maxilliped. Scale bars: $A=1 \mathrm{~mm}$; $-\mathrm{H}=0.1 \mathrm{~mm}$. 


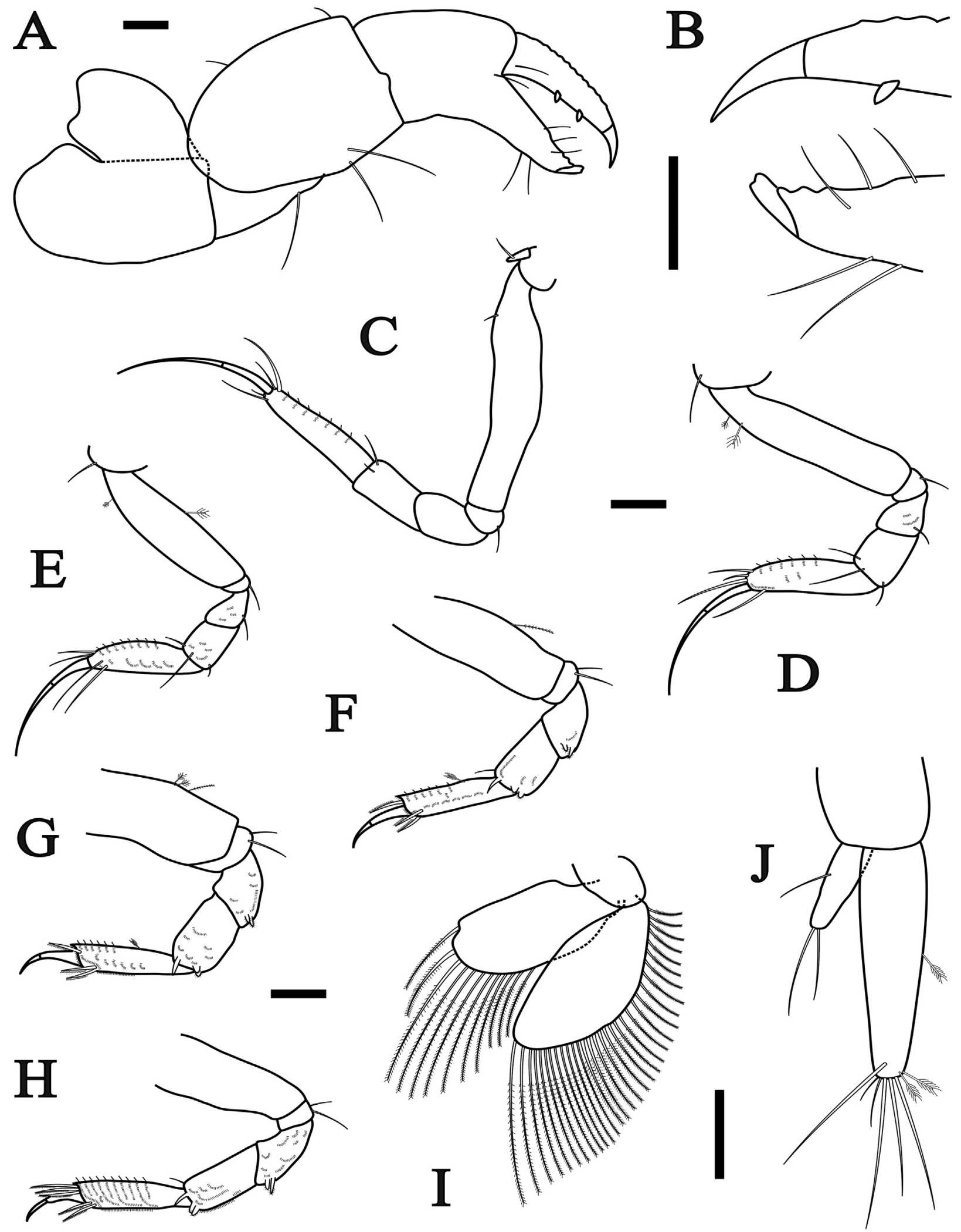

Figure 2. Tanaopsis bamberi sp. nov., female paratype, length $4.5 \mathrm{~mm}$, MNRJ 23402. A, cheliped, with B, detail of cheliped fixed finger; C, pereopod 1; D, pereopod 2; E, pereopod 3; F, pereopod 4; G, pereopod 5; H, pereopod 6; I, pleopod; J, uropod. Scale bars = $0.1 \mathrm{~mm}$. 
1.6 times as long as wide, with four inner distal plumose and two simple setae. Article 3 shortest, as long as wide, with two outer distal simple and one plumose seta. Article 4 slender, about 3.8 times as long as wide, with one aesthetasc and seven simple terminal setae.

Antenna (Fig. 1C) about 0.9 times as long as antennule. Article 1 broader than following articles, naked. Article 2 longer than article 3, about 1.3 times as long as wide, with one inner distal simple seta and one outer distal long simple seta. Article 3 as long as wide, with one outer distal simple seta. Article 4 narrow and longest, about 4.5 times as long as wide and 1.3 times longer than the last two articles together, with two subdistal plumose setae and three distal simple setae. Article 5 about 3.5 times as long as wide, longer than article 2, with one distal simple seta. Article 6 minute, with six terminal setae.

Mouthparts. Labrum not recovered. Mandibles (Fig. 1D, E) molar with distal margin crenulated and molar thin and tapering. Left mandible (Fig. 1D) incisor with rounded and inflated tip; lacinia mobilis well-developed, crenulated. Right mandible (Fig. 1E) incisor not bifurcated. Labium not recovered. Maxillule (Fig. 1F) endite bent at rightangle, with six simple apical setae, one significantly more robust than the others; palp with two distal long setae. Maxilla (Fig. 1G) rectangular. Maxilliped (Fig. 1H). Basis fused, with two long simple setae arising from tubercles on distal margin; endite not fused, each with one short simple seta distally; palp article 1 naked; article 2 with two inner and one outer plumose setae; article 3 trapezoidal, with four inner plumose setae; article 4 slender than the others, with five plumose terminal setae. Epignath not recovered.

Cheliped (Fig. 2A, B). Basis about 1.7 times as long as wide, shorter than carpus, attached to cephalothorax via large sclerite. Merus triangular, with one long simple seta ventrally. Carpus about 1.3 times as long as wide, no carpal shield, with two long simple ventral setae and two minute setae dorsally. Propodus about 1.8 times as long as wide, with one simple seta near dactylus insertion. Fixed finger having delicate bifid terminal spine, with three inner and two outer simple setae and inner margin with three smooth sub-terminal spines. Dactylus slightly longer than fixed finger, with weak crenulations on dorsal margin and with two spines ventrally.
Pereopod 1 (Fig. 2C). Coxa with prominent spur with simple seta. Basis long (about 5.6 times as long as wide), with one minute seta dorsally. Ischium with one ventral simple seta. Merus apparently naked, 1.1 times as long as wide. Carpus slightly longer than merus, with two simple setae distally. Propodus as long as merus and carpus combined, with four simple terminal setae and dorsal microtrichia. Dactylus more than half as long as unguis, combined longer than propodus.

Pereopod 2 (Fig. 2D). As pereopod 1 except coxa without prominent spur. Basis 4.75 times as long as wide, with two minute plumose setae dorsally. Merus as long as wide, with one distal simple seta. Carpus with three setae distally. Propodus shorter than merus and carpus combined, with row of microtrichia along outer margin.

Pereopod 3 (Fig. 2E). As pereopod 2 except basis 3.75 times as long as wide, with one ventral and one plumose seta dorsally. Propodus with row of microtrichia along outer margin.

Pereopod 4 (Fig. 2F). Stouter than pereopods 1-3. Coxa absent. Basis about 2.7 times as long as wide, with one plumose seta ventrally. Ischium short, with two simple setae ventrally. Merus 1.3 times as long as wide, with two long feathery distal spines. Carpus longer than merus, with one pointed and two round feathery spines and one distal simple seta. Propodus shorter than merus and carpus combined, with one plumose seta dorsally, three serrated spiniform terminal setae and row of microtrichia along outer margin. Dactylus 1.2 times as long as unguis.

Pereopod 5 (Fig. 2G). As pereopod 4 except basis 1.9 times as long as wide, with three plumose setae ventrally. Carpus with one pointed and two round feathery spines distally. Dactylus 1.5 times as long as unguis.

Pereopod 6 (Fig. 2H). As pereopod 4 except basis 2.3 times as long as wide, naked. Merus 1.5 times as long as wide. Propodus tipped by five serrated spiniform setae.

Pleopods (Fig. 2I). Well-developed. All pleopods similar in structure, with unequal rami. Basal article short, as long as wide. Endopod subrectangular with all setae distal, with one outer and eleven plumose inner setae. Exopod subovate with 32 plumose setae on entire outer margin; no gaps between the most proximal and the other setae. 
Uropod (Fig. 2J). Shorter than pleotelson. Basal article shorter than exopod, naked. Exopod uniarticulate, 0.4 times length of endopod, with one long simple seta medially and two apically. Endopod uniarticulate, with one medial plumose seta and two plumose and six simple terminal setae.

Remarks: The main differences between the species of Tanaopsis are shown in Tab. 2. Tanaopsis bamberi sp. nov. resembles $T$. boonwurrungi and $T$. canaipa recorded from Australia and T. kerguelenensis from Antarctica, particularly in these species having antennule long (article 1 more than twice as long as wide), pleotelson long and uropod endopod more than 2.5 times longer than exopod. However, $T$. bamberi sp. nov. has more stout body (4.9 times); maxilliped basis with seta longer than endite and arising from tubercles; maxilliped endite with seta short and subdistal and inner margin of cheliped fixed finger with three smooth sub-terminal spines.

Also, T. bamberisp. nov. is distinct from T. antarctica, T. chotkarakde, T. graciloides (Lilljeborg, 1864), T. oios, T. profunda, T. rawhitia and T. rugaris BłażewiczPaszkowycz et al., 2013 by the antennule article 2 rectangular and longest (about 2.7 times as long as wide) and the uropod endopod without segmentation or fusion line. The new species distinguish from T. cadieni, T. gallardoi and T. laticaudatus by the maxilliped endite having a short subdistal seta instead of being absent and the uropod endopod and exopod uniarticulate instead of biarticulate.

Moreover, T. curtus has carapace 1.1 times as long as the first three pereonites (T. bamberi has carapace 0.7 times as long as pereonites 1-3) and has maxilliped basis seta absent. Finally, $T$. bamberi sp. nov. is immediately distinguished from all of these taxa, except $T$. boonwurrungi and T. kerguelenensis, by its antennule article 1 and article 2 longest (about 2.75 and 1.6 times as long as wide, respectively) and both rami of uropod uniarticulate, without a fusion line.

Bathymetric distribution: 55.7-400 m.

\section{Tanaopsis brevicorpus sp. nov.}

(Figs. 3-6)

Material examined. Holotype. One adult non-ovigerous female, length $3.1 \mathrm{~mm}$ (MNRJ

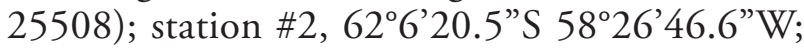
125 m; 7 December 2009. Paratype. One male, length $3.2 \mathrm{~mm}$ (MNRJ 25192); station Botany Point R3, 6205'42.1”S 58²0'44.5”W; $64.3 \mathrm{~m} ; 26$ January 2004. Three adults nonovigerous female (MNRJ 23401); length $2.8 \mathrm{~mm}$ (dissected); length $3.1 \mathrm{~mm}$; length $3.9 \mathrm{~mm}$ (only mouthparts dissected); station \#2, 62\%6'20.5”S $58^{\circ} 26^{\prime} 46.6^{\prime \prime W} ; 125$ m; 7 December 2009. Ten adult non-ovigerous females and five manca (MNRJ 23401); same locality. One adult nonovigerous female (MNRJ 23397); station Botany Point 1 R1, 6205'44.3"S 58²0'57.6”W; 55.7 m; 29 January 2005. One adult non-ovigerous female (MNRJ 23398); station Botany Point 1 R2, 62 05'44.1"S 58²0'58.1”W; $58.2 \mathrm{~m}$; 3 February 2005. Seven adult non-ovigerous female (MNRJ 23399); station Botany Point 2 R1, 6205'47.6”S 58²0’31.0”W; $58.6 \mathrm{~m}$; 9 February 2005. Eight adult non-ovigerous female (MNRJ 23403); station Botany Point 2 R2; 6205'47.3”S 58²0’31.4”W; 58.6 m; 5 December 2004. Eleven adult non-ovigerous females and two mancas (MNRJ 23400); station \#1, 6205'54.0”S 58²5'46,9”W; $120 \mathrm{~m} ; 7$ December 2009. One juvenile (MNRJ 24440); station \#1, 62 $09^{\circ} 52.4^{\prime \prime S} 58^{\circ} 23^{\prime} 43.5^{\circ} \mathrm{W} ; 500.8$ m; 2 December 2009.

Diagnosis. Female. Antennule first article 1.4 times as long as wide. Antenna with six articles, second and third articles with long spiniform setae subequal in length. Carapace as long as first three pereonites combined. Inner margin of cheliped fixed finger with one large sub-terminal spine. Both rami of uropod biarticulate (with fusion line) and endopod 2.1 times as long as exopod.

Etymology. Named after the body stout of the new species, with the lowest proportion length/ width among Tanaopsis species (4.1 times).

Description. Female paratype, $2.8 \mathrm{~mm}$ (dissected).

Body (Fig. 3A, B). Body about four times as long as wide. Cephalothorax triangular, 1.1 times as wide as long, as long as first three pereonites combined; with one lateral simple medial seta in each side; rostrum rounded; eyelobes absent. Pereonites. All pereonites with slightly rounded corners and with one seta in each lateral margin. Pereonite 1 shortest, about 0.25 as long as carapace. Pereonite 3 as long as pereonites 2 and 
6. Pereonite 5 longer than pereonite 3. Pereonite 4 longest. Pleon longer than $30 \%$ of body length. All pleonites with one lateral simple seta each. Pleonite 6 longest. Pleotelson about two last pleonites combined (about 30\% length of pleon), with four pairs of terminal simple setae. Apex pointed.

Antennule (Fig. 3C). About 0.9 times as long as cephalothorax. Article 1 longest but as short as the rest of antennule, about 1.45 times as long as wide, inner distal margin with six plumose and one long simple setae and one outer distal simple seta. Article 2 half length of article 1 , about 1.1 times as long as wide, with three inner distal plumose and two simple setae and one outer distal simple seta. Article 3 shortest, as long as wide, with one outer distal plumose and two simple setae. Article 4 slender, about 3.2 times as long as wide, with one aesthetasc and eight simple terminal setae.

Antenna (Fig. 3D). About 0.9 times as long as antennule, with six articles. Article 1 broader than following articles, naked. Article 2 as long as article 3 , about 1.3 times, with one outer distal long simple seta. Article 3 square, with one outer distal long simple seta. Article 4 longest, about 4.2 times as long as wide, and 1.3 times as long

Table 2. List of mainly diagnostic characters of all described species of Tanaopsis Sars, 1899 (based on female morphology). Abbreviations: A1 = antennule; art. = article; $\mathrm{mnd} .=$ mandibule; $\operatorname{mxp} .=$ maxilliped; pereon. = pereonite; prop. = length $/$ width; endop. = endopod; exop. = exopod. * Form of spiniform process on inner margin of cheliped fixed finger; ? Information not shown in articles cited.

\begin{tabular}{|c|c|c|c|c|c|c|c|c|}
\hline Species & $\begin{array}{c}\text { Tanaopsis } \\
\text { bamberi n. sp. }\end{array}$ & $\begin{array}{l}\text { T. brevicorpus } \\
\text { n. sp. }\end{array}$ & $\begin{array}{l}\text { T. antarctica } \\
\text { Lang, } 1967\end{array}$ & $\begin{array}{c}T . \\
\text { boonwurrungi } \\
\text { Błażewicz- } \\
\text { Paszkowycz } \\
\text { and Bamber, } \\
2012\end{array}$ & $\begin{array}{c}\text { T. cadieni Sieg } \\
\text { and Dojiri, } \\
1991\end{array}$ & $\begin{array}{c}\text { T. canaipa } \\
\text { Bamber, } 2008\end{array}$ & $\begin{array}{l}\text { T. chotkarakde } \\
\text { Bird and } \\
\text { Bamber, } 2000\end{array}$ & $\begin{array}{c}\text { T. curtus } \\
\text { Kudinova- } \\
\text { Pasternak, } \\
1984\end{array}$ \\
\hline Type locality & $\begin{array}{l}\text { Admiralty Bay } \\
\text { (Antarctica) }\end{array}$ & $\begin{array}{c}\text { Admiralty Bay } \\
\text { (Antarctica) }\end{array}$ & $\begin{array}{c}\text { South Georgia } \\
\text { Island } \\
\text { (Antarctica) }\end{array}$ & $\begin{array}{l}\text { Western Port } \\
\text { (Australia) }\end{array}$ & $\begin{array}{c}\text { Santa Catalina } \\
\text { Island } \\
\text { (California) }\end{array}$ & $\begin{array}{l}\text { Moreton Bay } \\
\text { (Australia) }\end{array}$ & $\begin{array}{l}\text { Tai Tam Bay } \\
\text { (Hong Kong) }\end{array}$ & Sea of Japan \\
\hline Body prop. & $4.9 \mathrm{x}$ & $4.1 \mathrm{x}$ & $?$ & $8.7 x$ & $5.5 x$ & $8.75 \mathrm{x}$ & $6.7 x$ & $4.5 x$ \\
\hline $\begin{array}{l}\text { Prop. A1 to } \\
\text { carapace }\end{array}$ & As long as & $0.9 \mathrm{x}$ & ? & $0.85 \mathrm{x}$ & $0.8 \mathrm{x}$ & $0.6 \mathrm{x}$ & $0.8 \mathrm{x}$ & ? \\
\hline $\begin{array}{l}\text { Prop. of A1 } \\
1 \text { st art. }\end{array}$ & $2.75 x$ & $1.45 \mathrm{x}$ & $1.3 \mathrm{x}$ & $2.1 \mathrm{x}$ & $1.5 \mathrm{x}$ & $2.0 \mathrm{x}$ & $1.8 \mathrm{x}$ & $2.1 \mathrm{x}$ \\
\hline $\begin{array}{l}\text { Prop. of A1 } \\
2 \text { nd art. }\end{array}$ & $1.6 \mathrm{x}$ & $1.1 \mathrm{x}$ & $1.0 \mathrm{x}$ & $1.0 \mathrm{x}$ & $1.15 \mathrm{x}$ & $1.2 \mathrm{x}$ & $1.4 \mathrm{x}$ & $1.0 \mathrm{x}$ \\
\hline Mnd. molar & Pointed & Pointed & Pointed & $\begin{array}{l}\text { Without } \\
\text { molar }\end{array}$ & Pointed & Pointed & $\begin{array}{l}\text { Without } \\
\text { molar }\end{array}$ & Pointed \\
\hline Mxp. basis seta & $\begin{array}{l}\text { Longer than } \\
\text { endite, arising } \\
\text { from tubercles }\end{array}$ & $\begin{array}{l}\text { Longer than } \\
\text { endite }\end{array}$ & $\begin{array}{c}\text { Longer than } \\
\text { endite }\end{array}$ & $\begin{array}{c}\text { Shorter than } \\
\text { endite }\end{array}$ & $\begin{array}{l}\text { Much longer } \\
\text { than endite }\end{array}$ & $\begin{array}{c}\text { Shorter than } \\
\text { endite }\end{array}$ & $\begin{array}{c}\text { Longer than } \\
\text { endite }\end{array}$ & Absent \\
\hline $\begin{array}{l}\text { Mxp. endite } \\
\text { seta }\end{array}$ & $\begin{array}{l}\text { Short; } \\
\text { subdistal }\end{array}$ & $\begin{array}{l}\text { Short, } \\
\text { arising from } \\
\text { tubercles; } \\
\text { subdistal }\end{array}$ & $\begin{array}{c}\text { Short, on } \\
\text { distal margin }\end{array}$ & Absent & Absent & $\begin{array}{c}\text { Short, on } \\
\text { distal margin }\end{array}$ & $\begin{array}{c}\text { Short, on } \\
\text { distal margin }\end{array}$ & ? \\
\hline $\begin{array}{l}\text { Prop. carapace/ } \\
\text { pereon. 1-3 }\end{array}$ & $0.7 x$ & As long as & ? & $0.5 x$ & $0.7 x$ & $0.75 x$ & $0.6 \mathrm{x}$ & $1.15 \mathrm{x}$ \\
\hline $\begin{array}{l}\text { Pereon. 1-6 } \\
\text { width/length. }\end{array}$ & $\begin{array}{c}3.5 ; 2.1 ; 1.85 \\
1.3 ; 1.56 \\
2.4 \mathrm{x}\end{array}$ & $\begin{array}{c}4.6 ; 3.4 ; 2.9 ; \\
1.9 ; 2.13 ; \\
3.6 \mathrm{x}\end{array}$ & ? & $\begin{array}{l}2.3 ; 1.4 ; 1.0 \\
0.8 ; 0.9 ; 1.2 \mathrm{x}\end{array}$ & $\begin{array}{l}3.8 ; 2.6 ; 1.6 ; \\
1.3 ; 1.3 ; 1.6 \mathrm{x}\end{array}$ & $\begin{array}{l}2.2 ; 1.5 ; 1.5 ; \\
0.9 ; 0.9 ; 1.2 \mathrm{x}\end{array}$ & $\begin{array}{l}3.2 ; 1.3 ; 1.5 ; \\
1.2 ; 1.5 ; 1.5 \mathrm{x}\end{array}$ & $\begin{array}{l}5.4 ; 3.3 ; 2.4 ; \\
1.9 ; 1.8 ; 3.5 \mathrm{x}\end{array}$ \\
\hline $\begin{array}{l}\text { Cheliped } \\
\text { spine* }^{*}\end{array}$ & $\begin{array}{l}3 \text { smooth } \\
\text { spines }\end{array}$ & 1 large spine & $\begin{array}{c}2 \text { spines ( } 1 \\
\text { large; } 1 \text { small) }\end{array}$ & 2 spines & 1 large spine & 2 spines & 2 spines & ? \\
\hline Pleotelson & $1.7 \mathrm{x}$ & $2.35 \mathrm{x}$ & $2.0 \mathrm{x}$ & $1.7 \mathrm{x}$ & $1.4 \mathrm{x}$ & $1.75 \mathrm{x}$ & $1.9 \mathrm{x}$ & $1.9 \mathrm{x}$ \\
\hline $\begin{array}{l}\text { Prop. uropod/ } \\
\text { pleotelson }\end{array}$ & $0.85 \mathrm{x}$ & $0.8 \mathrm{x}$ & $0.8 \mathrm{x}$ & $0.7 x$ & $0.8 \mathrm{x}$ & $0.6 \mathrm{x}$ & $0.75 \mathrm{x}$ & $?$ \\
\hline $\begin{array}{l}\text { Uropod } \\
\text { endopod }\end{array}$ & Uniarticulate & $\begin{array}{l}\text { Biarticulate } \\
\text { (fusion line) }\end{array}$ & Biarticulate & Uniarticulate & $\begin{array}{l}\text { Biarticulate } \\
\text { (fusion line) }\end{array}$ & Biarticulate & $\begin{array}{l}\text { Biarticulate } \\
\text { (fusion line) }\end{array}$ & Biarticulate \\
\hline Uropod exopod & Uniarticulate & $\begin{array}{l}\text { Biarticulate } \\
\text { (fusion line) }\end{array}$ & Biarticulate & Uniarticulate & Biarticulate & Biarticulate & Uniarticulate & Biarticulate \\
\hline $\begin{array}{l}\text { Uropod } \\
\text { endop./exop. }\end{array}$ & $2.55 \mathrm{x}$ & $2.1 \mathrm{x}$ & $2.1 \mathrm{x}$ & $2.5 \mathrm{x}$ & $1.8 \mathrm{x}$ & $2.6 \mathrm{x}$ & $2.3 \mathrm{x}$ & ? \\
\hline
\end{tabular}


Table 2. (Cont.)

\begin{tabular}{|c|c|c|c|c|c|c|c|c|}
\hline Species & $\begin{array}{c}\text { T. gallardoi } \\
\text { (Shiino, 1970) }\end{array}$ & $\begin{array}{l}\text { T. graciloides } \\
\text { (Lilljeborg, } \\
1864 \text { ) }\end{array}$ & $\begin{array}{c}T . \\
\text { kerguelenensis } \\
\text { Shiino, } 1978\end{array}$ & $\begin{array}{l}\text { T. laticaudatus } \\
\text { (Sars, 1882) }\end{array}$ & $\begin{array}{c}\text { T. oios } \\
\text { Btażewicz- } \\
\text { Paszkowycz } \\
\text { and Bamber, } \\
2012\end{array}$ & $\begin{array}{l}\text { T. profunda } \\
\text { Lang, } 1967\end{array}$ & $\begin{array}{l}\text { T. rawhitia } \\
\text { Bird, } 2011\end{array}$ & $\begin{array}{c}\text { T. rugaris } \\
\text { Błażewicz- } \\
\text { Paszkowycz et } \\
\text { al.., } 2013\end{array}$ \\
\hline Type locality & $\begin{array}{l}\text { Chile Bay } \\
\text { (Antarctica) }\end{array}$ & $\begin{array}{l}\text { Fjord Gullmar } \\
\text { (Sweden) }\end{array}$ & $\begin{array}{l}\text { Kerguelen } \\
\text { Islands } \\
\text { (Antarctica) }\end{array}$ & $\begin{array}{c}\text { Norway } \\
\text { and Naples } \\
\text { (Mediterranean) }\end{array}$ & $\begin{array}{l}\text { Bass Strait } \\
\text { (Australia) }\end{array}$ & Panama Basin & $\begin{array}{l}\text { Armer's } \\
\text { Bay (New } \\
\text { Zealand) }\end{array}$ & Sea of Japan \\
\hline Body prop. & $5.2 \mathrm{x}$ & $7.2 \mathrm{x}$ & $5.7 \mathrm{x}$ & $6.0 \mathrm{x}$ & ? & $?$ & $7.5 \mathrm{x}$ & ? \\
\hline $\begin{array}{l}\text { Prop. A1 to } \\
\text { carapace }\end{array}$ & As long as & $0.8 \mathrm{x}$ & As long as & $0.9 \mathrm{x}$ & ? & ? & $0.95 x$ & $0.9 \mathrm{x}$ \\
\hline $\begin{array}{l}\text { Prop. of A1 } \\
1 \text { st art. }\end{array}$ & $1.8 \mathrm{x}$ & $2.1 \mathrm{x}$ & $2.6 \mathrm{x}$ & $1.75 \mathrm{x}$ & $1.9 \mathrm{x}$ & $1.7 \mathrm{x}$ & $1.5 \mathrm{x}$ & $1.9 \mathrm{x}$ \\
\hline $\begin{array}{l}\text { Prop. of A1 } \\
2 \text { nd art. }\end{array}$ & $1.1 \mathrm{x}$ & $1.2 \mathrm{x}$ & $1.9 \mathrm{x}$ & $1.2 \mathrm{x}$ & $1.25 \mathrm{x}$ & $1.3 \mathrm{x}$ & $1.1 \mathrm{x}$ & $1.0 \mathrm{x}$ \\
\hline Mnd. molar & Pointed & Without molar & Without mnd. & Without molar & Without mnd. & Pointed & Pointed & Without mnd. \\
\hline Mxp. basis seta & $\begin{array}{l}\text { Much longer } \\
\text { than endite }\end{array}$ & $\begin{array}{l}\text { Longer than } \\
\text { endite }\end{array}$ & Absent & $\begin{array}{l}\text { Longer than } \\
\text { endite }\end{array}$ & $\begin{array}{l}\text { Longer than } \\
\text { endite }\end{array}$ & $\begin{array}{l}\text { Much shorter } \\
\text { than endite }\end{array}$ & $\begin{array}{c}\text { Much longer } \\
\text { than endite }\end{array}$ & ? \\
\hline $\begin{array}{l}\text { Mxp. endite } \\
\text { seta }\end{array}$ & Absent & $\begin{array}{c}\text { Short, on } \\
\text { distal margin }\end{array}$ & $\begin{array}{l}\text { Short; } \\
\text { subdistal }\end{array}$ & Absent & $\begin{array}{l}\text { Short; } \\
\text { subdistal }\end{array}$ & $\begin{array}{l}\text { Short; } \\
\text { subdistal }\end{array}$ & $\begin{array}{l}\text { Short, on } \\
\text { distal margin }\end{array}$ & ? \\
\hline $\begin{array}{l}\text { Prop. carapace/ } \\
\text { pereon. } 1-3\end{array}$ & $0.6 \mathrm{x}$ & $0.7 \mathrm{x}$ & $0.8 \mathrm{x}$ & $0.75 \mathrm{x}$ & ? & ? & $0.6 \mathrm{x}$ & $0.5 \mathrm{x}$ \\
\hline $\begin{array}{l}\text { Pereon. 1-6 } \\
\text { prop. }\end{array}$ & $\begin{array}{c}2.9 ; 2.55 ; 1.9 ; \\
1.5 ; 1.5 ; 2.3 \mathrm{x}\end{array}$ & $\begin{array}{l}2.8 ; 2.2 ; 1.3 ; \\
1.2 ; 1.1 ; 1.4 \mathrm{x}\end{array}$ & $\begin{array}{l}3.1 ; 1.8 ; 2.1 ; \\
1.6 ; 1.9 ; 2.2 \mathrm{x}\end{array}$ & $\begin{array}{c}3.6 ; 2.4 ; 1.5 ; \\
1.25 ; 1.2 ; \\
1.5 \mathrm{x}\end{array}$ & ? & ? & $\begin{array}{c}3.2 ; 1.7 ; 1.5 ; \\
1.1 ; 1.0 ; \\
1.55 \mathrm{x}\end{array}$ & ? \\
\hline $\begin{array}{l}\text { Cheliped } \\
\text { spine* }\end{array}$ & 1 large spine & $\begin{array}{c}2 \text { spines (1 } \\
\text { large; } 1 \text { small) }\end{array}$ & 2 spines & 1 large spine & 2 spines & $\begin{array}{c}2 \text { spines (1 } \\
\text { large; } 1 \text { small) }\end{array}$ & 2 spines & 2 spines \\
\hline Pleotelson & $2.1 \mathrm{x}$ & $1.8 \mathrm{x}$ & $1.45 \mathrm{x}$ & $1.7 \mathrm{x}$ & ? & $2.3 \mathrm{x}$ & $1.9 \mathrm{x}$ & ? \\
\hline $\begin{array}{l}\text { Prop. uropod/ } \\
\text { pleotelson }\end{array}$ & $0.9 \mathrm{x}$ & $0.6 \mathrm{x}$ & $0.5 \mathrm{x}$ & $0.7 \mathrm{x}$ & ? & $1.0 \mathrm{x}$ & $0.9 \mathrm{x}$ & $0.6 \mathrm{x}$ \\
\hline $\begin{array}{l}\text { Uropod } \\
\text { endopod }\end{array}$ & Biarticulate & Biarticulate & Uniarticulate & Biarticulate & Biarticulate & Biarticulate & $\begin{array}{l}\text { Biarticulate } \\
\text { (fusion line) }\end{array}$ & $\begin{array}{l}\text { Biarticulate } \\
\text { (fusion line) }\end{array}$ \\
\hline Uropod exopod & Biarticulate & Biarticulate & Uniarticulate & Biarticulate & Biarticulate & Biarticulate & Uniarticulate & Biarticulate \\
\hline $\begin{array}{l}\text { Uropod } \\
\text { endop./exop. }\end{array}$ & $2.1 \mathrm{x}$ & $2.0 \mathrm{x}$ & $3.5 \mathrm{x}$ & $2.0 \mathrm{x}$ & $1.9 \mathrm{x}$ & $2.3 \mathrm{x}$ & $1.85 \mathrm{x}$ & $2.2 \mathrm{x}$ \\
\hline
\end{tabular}

as the last two articles, one medial and five distal plumose setae and three distal simple setae. Article 5 three times as long as wide, longer than article 3 , with one single distal seta as long as fourth article. Article 6 minute, with six simple terminal setae.

Mouthparts. Labrum not recovered. Labium (Fig. 3E) with outer distal process, with few setules. Mandibles (Fig. 3F, G) with distal margins crenulated and molar thin and tapering. Left mandible (Fig. 3F) incisor with rounded and bloated tip; lacinia mobilis well-developed, with crenulations. Right mandible (Fig. 3G) incisor not bifurcated, weakly calcified. Maxillule (Fig. $4 \mathrm{~A}, \mathrm{~B})$ endite with several fine setules on lateral margins, six apical pinnate setae; palp with two distal simple setae. Maxilla (Fig. 4C) triangular, naked. Maxilliped (Fig. 4D) basis partly fused, with two long simple setae on distal margin; endite not fused, with two short simple setae arising from tubercles; palp article 1 naked; article 2 with two inner and one outer simple setae and two medial rows of minute setules; article 3 trapezoidal, with four inner simple setae and two distal rows of minute setules; article 4 slender than the others, with six simple terminal setae and four distal rows of minute setae. Epignath not recovered.

Cheliped (Fig. 4E, F). Basis about 1.6 times as long as wide, as long as carpus, attached to cephalothorax via large sclerite, with dorsodistal simple seta. Merus triangular, with one long simple seta ventrally. Carpus about 1.3 times as long as wide, no carpal shield, with two long simple ventral setae and two minute setae dorsally. Propodus two times as long as wide, with five simple setae and two groups of minute setae near dactylus insertion. Fixed finger having delicate bifid terminal spine, with three inner and two outer simple setae and inner margin with one 

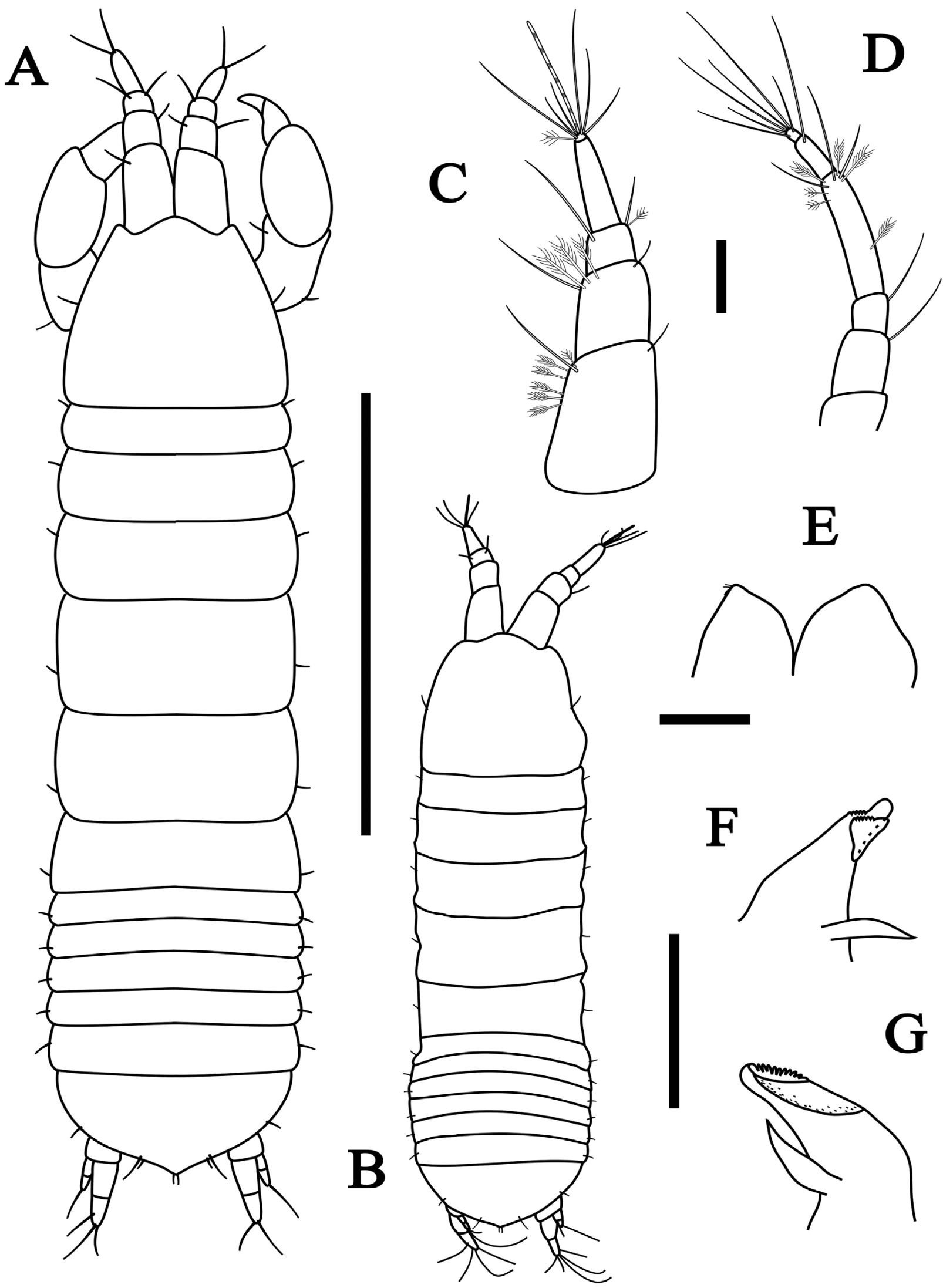

Figure 3. Tanaopsis brevicorpus sp. nov., female paratype, length $2.8 \mathrm{~mm}$, MNRJ 23401. A, female dorsal view; B, manca dorsal view, length $1.3 \mathrm{~mm}$; C, antennule, D, antenna, E, labium, F, left mandible, G, right mandible. Scale bars: A-B = $1 \mathrm{~mm}$; C-G = $0.1 \mathrm{~mm}$. 


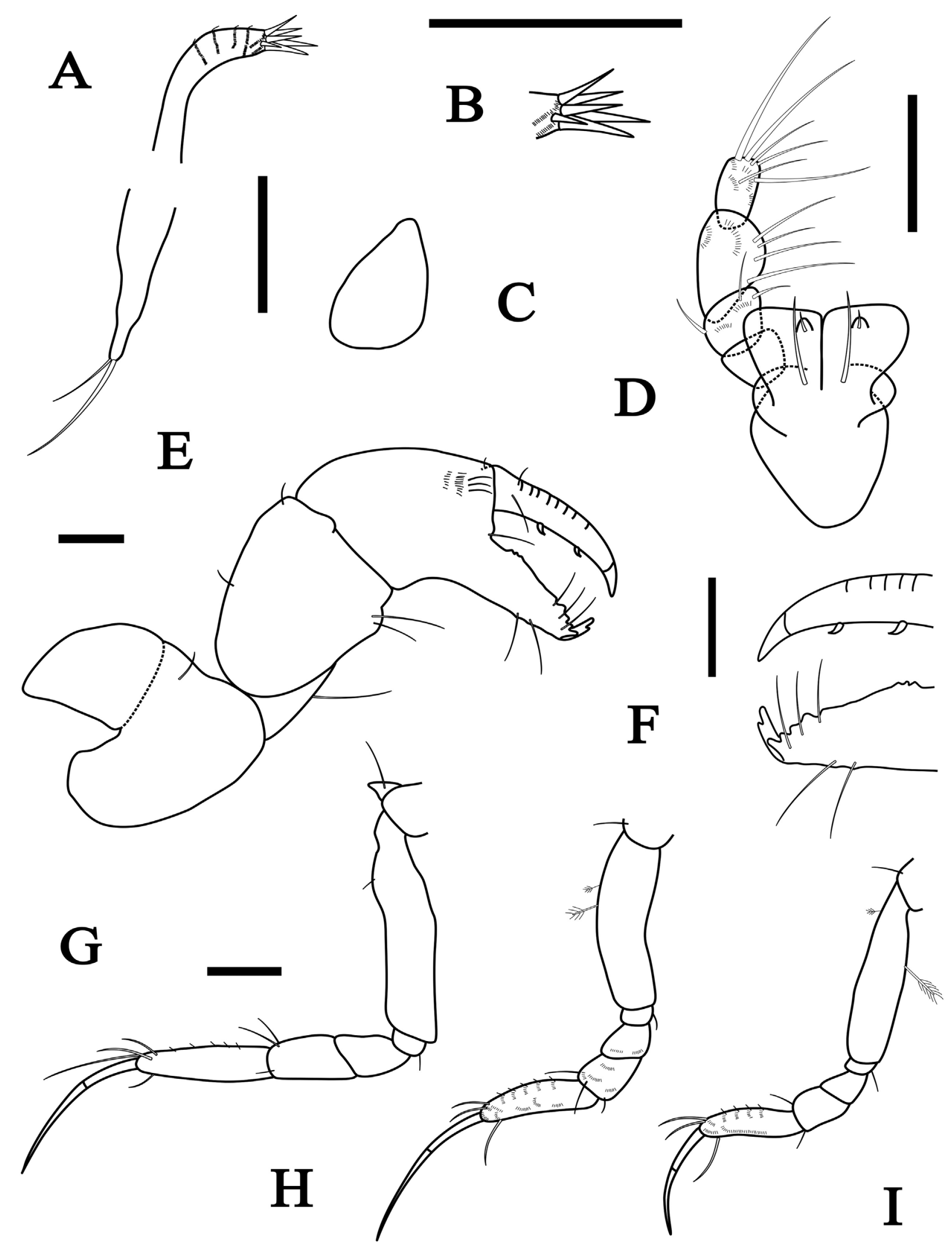

Figure 4. Tanaopsis brevicorpus sp. nov., female paratype, length $2.8 \mathrm{~mm}$, MNRJ 23401. A, maxillule, with B, detail of maxillule; C, maxilla; D, maxilliped; E, cheliped, with, F, detail of cheliped fixed finger; G, pereopod 1; H, pereopod 2; I, pereopod 3 . Scale bars $=0.1 \mathrm{~mm}$. 


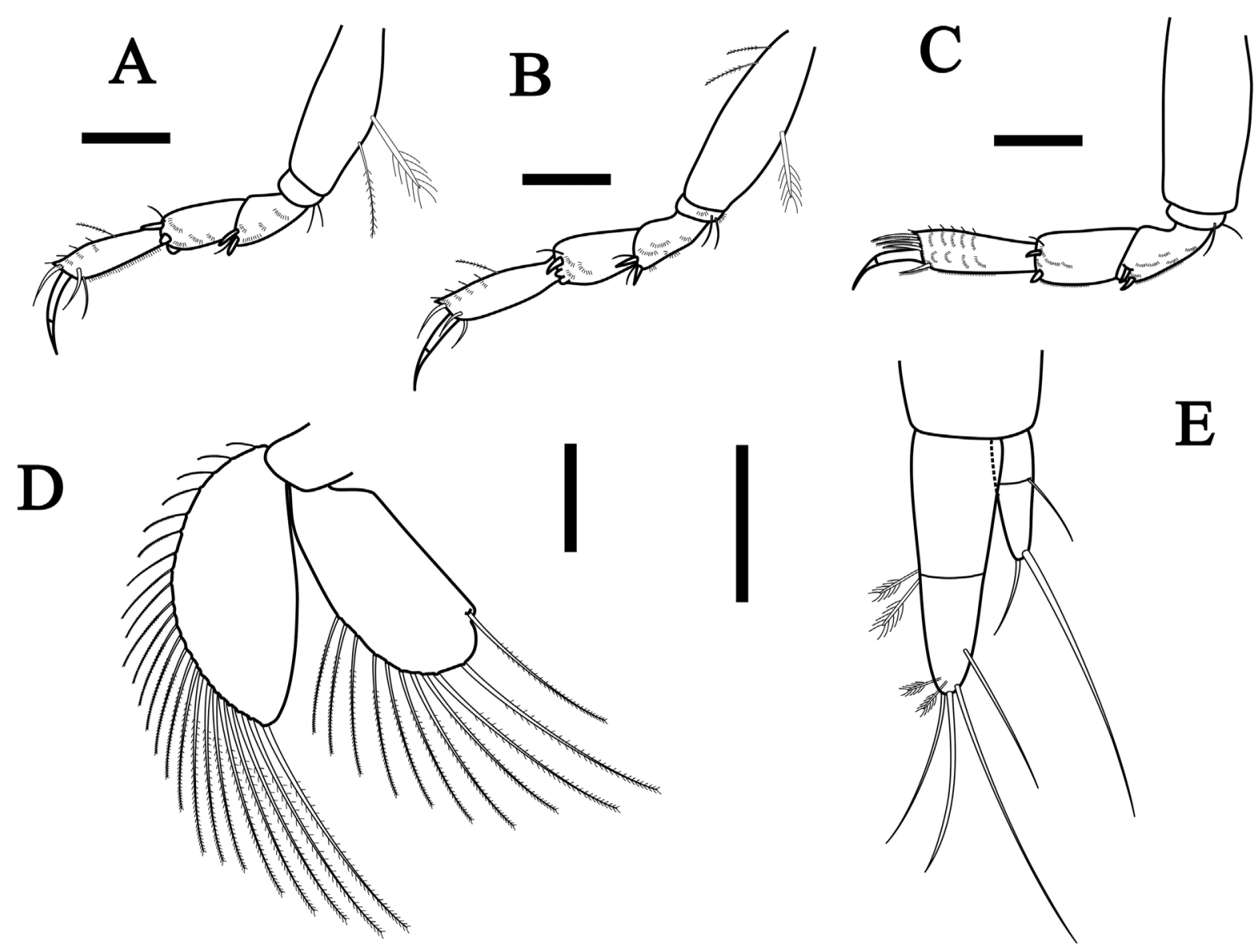

Figure 5. Tanaopsis brevicorpus sp. nov., female paratype, length 2.8, mm, MNRJ 23401. A, pereopod 4; B, pereopod 5; C, pereopod 6; D, pleopod; E, uropod. Scale bars $=0.1 \mathrm{~mm}$.

large sub-terminal spine. Dactylus as long as fixed finger, with crests on outer margin, two simple setae and two spines ventrally.

Pereopod 1 (Fig. 4G). Coxa present, with prominent spur with long simple seta. Basis long (about 4.7 times as long as wide), with one minute dorsal seta. Ischium short, with one ventral simple seta. Merus naked, 1.3 times as long as wide. Carpus longer than merus, with three simple setae distally. Propodus as long as merus and carpus combined, with row of minute setae along outer margin and four spiniform terminal setae. Dactylus half as long as unguis. Dactylus and unguis combined longer than propodus.

Pereopod 2 (Fig. 4H). As pereopod 1 except coxa without prominent spur. Basis 3.4 times as long as wide, with two plumose setae dorsally. Merus as long as wide, with medial rows of minute setules. Propodus longer than merus and carpus combined, with three spiniform terminal setae and medial rows of minute setules. Dactylus less than half as long as unguis.

Pereopod 3 (Fig. 4I). As pereopod 1 except coxa without prominent spur, basis 4.4 times as long as wide, with one inner and one outer plumose seta. Carpus with two distal simple setae.

Pereopod 4 (Fig. 5A). Shorter than pereopod 1-3. Coxa absent. Basis about 2.4 times as long as wide, with one long plumose seta ventrally. Ischium short, with two simple setae ventrally. Merus 1.3 times as long as wide, with medial rows of minute setules and two pointed processes and one simple seta distally. Carpus longer than merus, with one pointed process, two tubercles and one simple seta distally. Propodus shorter than merus and carpus combined, with one long plumose seta dorsally, three spiniform terminal setae and row of minute setae along outer and inner margins. Dactylus about two times as long as unguis.

Pereopod 5 (Fig. 5B). As pereopod 4 except basis 3 times as long as wide, with two inner and one outer plumose setae. Merus twice as long as 


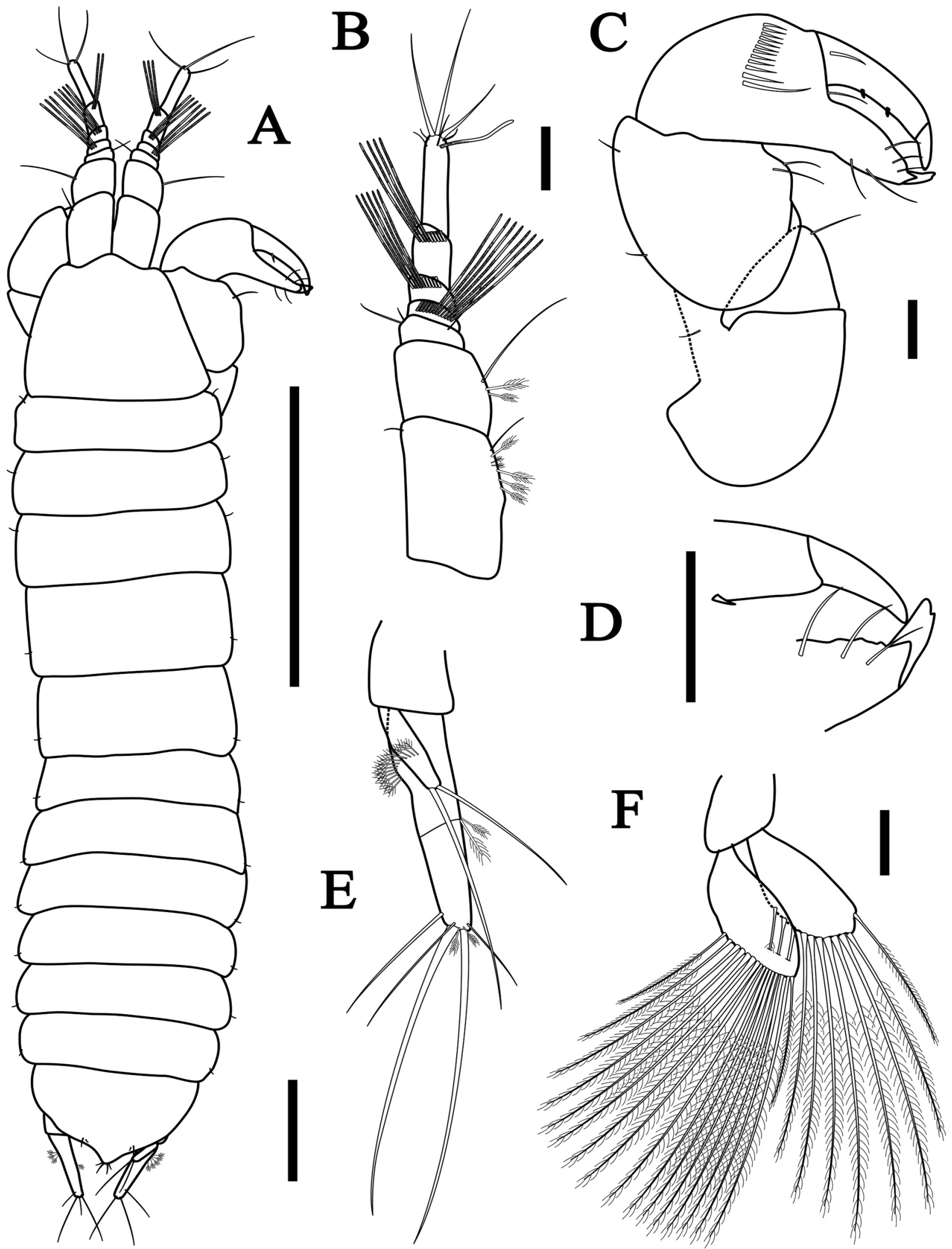

Figure 6. Tanaopsis brevicorpus sp. nov., male paratype, length $3.2 \mathrm{~mm}$, MNRJ 25192. A, dorsal view; B, antennules; C, cheliped, with D, detail of cheliped fixed finger; E, uropod; F, pleopod. Scale bars: $A=1 \mathrm{~mm} ; \mathrm{B}-\mathrm{F}=0.1 \mathrm{~mm}$. 
wide, with two simple setae distally. Dactylus about as long as unguis.

Pereopod 6 (Fig. 5C). As pereopod 4 except basis 2.1 times as long as wide, naked. Merus with two pointed processes distally. Carpus with two pointed processes and one simple setae distally. Propodus tipped by five serrated spiniform setae. Dactylus as long as unguis.

Pleopods (Fig. 5D). Well-developed. All pleopods similar in structure, with unequal rami. Basal article short, 1.1 times as wide as long. Endopod subrectangular with all setae distal, with one outer and ten plumose inner setae. Exopod sub-ovate with 24 plumose setae on entire posterior margin; no gaps between the most proximal and other setae.

Uropod (Fig. 5E). Shorter than pleotelson. Basal article shorter than exopod, naked. Exopod biarticulate (with fusion line), half of endopod, with one simple medial seta and two long setae apically. Endopod biarticulate (with fusion line), with two plumose setae medially, one simple subdistal seta and tipped by two plumose and three long simple terminal setae.

Manca paratype $1.3 \mathrm{~mm}$ (Fig. 3B). Smaller than female and similar in morphology. Antennule, antenna and uropod have the same number of article as adults and cheliped has the same structure (even the tip of fixed finger) as full developed females, been the only difference between them: pereonites and pleotelson slightly wider than in adults.

Male paratype, $3.2 \mathrm{~mm}$. Not dissected. Sexual dimorphism present. Feeding male. Body (Fig. 6A) about 5.5 times as long as wide. Cephalothorax triangular, 1.2 times as wide as long; rostrum rounded; eyelobes absent. Pereon. Pereonites lesser than female; pereonite 1 shortest, about 0.3 as long as carapace. Pereonite 4 longest, 2.0 times as wide as long. Pleon. Pleonites developed about $30 \%$ of body length. Antennule (Fig. 6B) 4.75 times as long as wide, as long as cephalothorax, with seven articles; article 1 longest, inner distal margin with six plumose and one long simple setae and one outer distal simple seta; article 2 half length of article 1, with two inner medial plumose and one long simple setae; article 3 short, with one inner and two outer distal simple setae; articles 4-6 densely packed with aesthetascs; article 7 slender, with one aesthetasc, four short and three long simple terminal setae. Maxilliped developed, not recovered. Cheliped (Fig. 6C, D) same as in female. Uropod (Fig. 6E) exopod uniarticulate, less than half of endopod, with two long setae on the tip. Endopod biarticulate (with fusion line), with twelve plumose setae proximally, two plumose setae medially and tipped by two plumose and five simple terminal setae. Pleopods (Fig. 6F) well-developed. Endopod with one outer and ten plumose inner setae. Exopod with 17 plumose inner setae; no gaps between the most proximal and other setae.

Remarks. Tanaopsis is a morphologically conservative genus (Bird and Bamber, 2000), being reported variations only by Lang (1967) in pleon shape of $T$. graciloides females. Thus, the few variations found in species are considered for this to be new for science.

Tanaopsis brevicorpus sp. nov. resembles $T$. antarctica, T. cadieni, T. chotkarakde, T. laticaudatus and T. profunda, however differs from these species by having carapace about as long as first three pereonites; pleotelson short (2.35 times as long as wide); proportions of uropod and pleotelson (0.85 times) and exopod of uropod with fusion line.

The new species is distinct from T. boonwurrungi, T. canaipa, T. graciloides, T. kerguelenensis, T. oios, $T$. rawhitia and $T$. rugaris by maxilliped endite with short seta and arising from tubercles; inner margin of cheliped fixed finger with one large spine; pleotelson short and uropod both rami with fusion line. Tanaopsis curtus has the carapace longer than pereonites 1-3 (1.15 times) and maxilliped basis seta absent. Furthermore, in comparison with T. gallardoi, T. brevicorpus sp. nov. has less slender antennule article 2, maxilla triangular instead of with two lobes, both rami of uropod having fusion line (semi-fused articles) and uropod about half length of pleotelson in place of subequal.

\section{Bathymetric distribution. 55-500 m.}

Sieg and Dojiri (1991) gave a key to the species then known. In this key were included T. laticaudatus as a synonym of T. graciloides and excluded $T$. gallardo $i$ (before as Leptognathia gallardoi). A key to all species of the genus Tanaopsis up to now (16 species including T. bamberi sp. nov. and T. brevicorpus sp. nov.) is given. 
Key to species of Tanaopsis Sars, 1899 (females only)

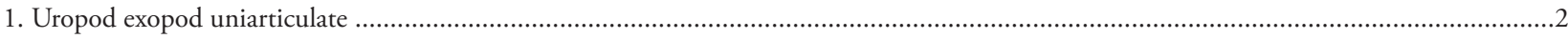

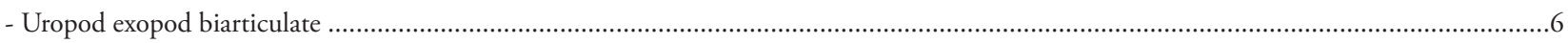

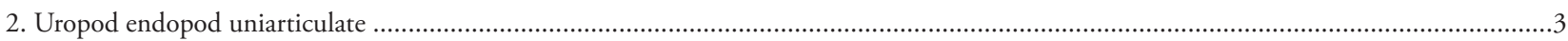

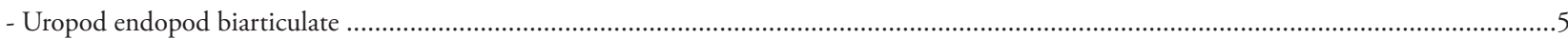

3. Pereonites 4 and 5 longer than wide; uropod exopod shorter than half length of endopod ................................................................ 4

- Pereonites 4 and 5 wider than long; uropod exopod distinctly shorter than first endopod article................................... Tanaopsis kerguelenensis Shiino, 1978

4. Body slender, more than eight times as long as wide; presence of small distal article on the antennule (minute article); species from Australia ..

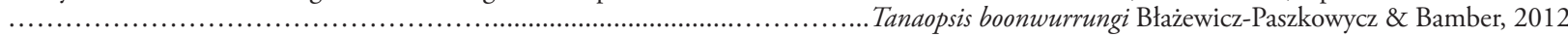

- Body stout, about five times as long as wide; antennule without small distal article (minute article); species from Antarctica ......................

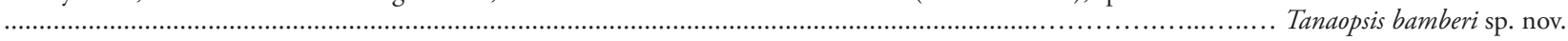

5. Pereonite 5 as long as wide; antenna article 2 square, about as long as wide; uropod exopod longer than half length of endopod; presence of small distal article on the antennule (minute article); species from New Zealand.....

..Tanaopsis rawhitia Bird, 2011

- Pereonite 5 wider than long; antenna article 2 rectangular, longer than wide; uropod exopod shorter than half length of endopod; pereopod 1 much larger than other pereopods; species from Hong Kong..... Tanaopsis chotkarakde Bird \& Bamber, 2000

6. Uropod endopod with distinct articles 7

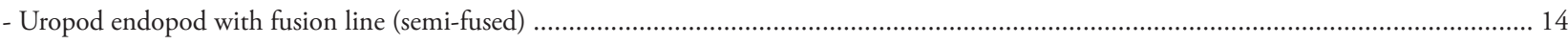

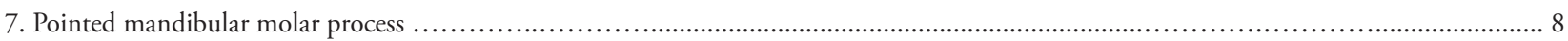

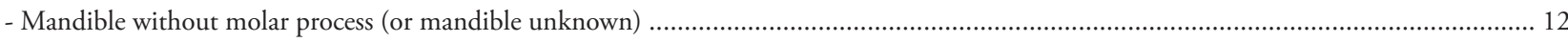

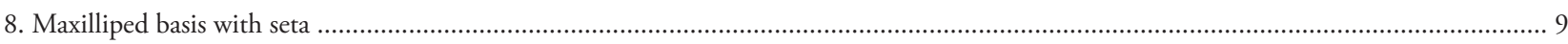

- Maxilliped basis seta absent; very compact pereonites; carapace longer than pereonites 1-3 combined; species from Japan ................................ …......................................................................................................................... Tanaopsis curtus Kudinova-Pasternak, 1984

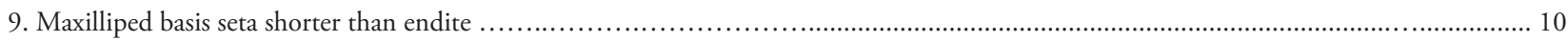

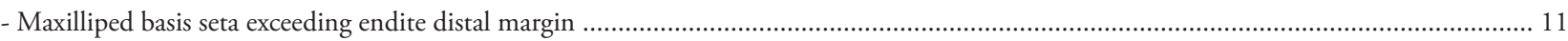

10. Antennule article 1 twice as long as wide; inner margin of cheliped fixed finger with two sub-terminal spines of same size (inner bifurcate claw and outer trifurcate claw); pleotelson less than twice as long as wide; uropod about half length of pleotelson; species from Australia .............

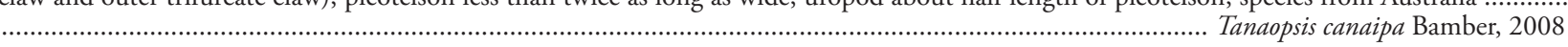

- Antennule article 1 less than twice as long as wide; inner margin of cheliped fixed finger with two sub-terminal spines of different sizes; pleotelson more than twice as long as wide; uropod as long as pleotelson; maxilliped basis seta distinctly shorter than endite (seta extends to about half of endite); species from Panama Basin .....

.. Tanaopsis profunda Lang, 1967

11. Maxilliped endite with short seta; inner margin of cheliped fixed finger with two sub-terminal spines

Tanaopsis antarctica Lang, 1967

- Maxilliped endite without seta; inner margin of cheliped fixed finger with one large spine; maxilliped basis seta very long, almost reaching palp article 3

Tanaopsis gallardoi (Shiino, 1970)

12. Two sub-terminal spines on inner margin of cheliped fixed finger.

- One large sub-terminal spine on inner margin of cheliped fixed finger; species from Norway and Mediterranean

Tanaopsis laticaudatus (Sars, 1882)

13. Spines on inner margin of cheliped fixed finger of same size; maxilliped endite with one subdistal short seta; species from Australia .........

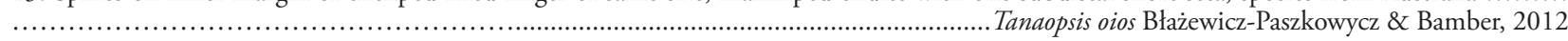

- Spines on inner margin of cheliped fixed finger of different sizes (1 large and 1 small); maxilliped endite with one distal short seta; species from Sweden Tanaopsis graciloides (Lilljeborg, 1864)

14. Inner margin of cheliped fixed finger with one large sub-terminal spine; antennule without minute article; antenna article 4 without fusion line

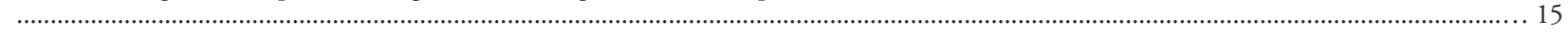

- Inner margin of cheliped fixed finger with two spines; uropod exopod as long as first endopod article; presence of a small distal article on the antennule (minute article); antenna article 4 with fusion line; cheliped dactylus rugosity not restricted to the distal half of it; species from Japan .......................................................................................................... Tanaopsis rugaris Błażewicz-Paszkowycz et al., 2013

15. Uropod exopod large, longer than first endopod article; maxilliped endite seta absent; pleotelson about 1.5 times longer than wide; species from California Tanaopsis cadieni Sieg \& Dojiri, 1991

- Uropod exopod as long as first endopod article; maxilliped endite seta short, arising from tubercles; pleotelson more than twice longer than wide. 


\section{ACKNOWLEDGEMENTS}

The authors would like to thank Dr. Lúcia de Siqueira Campos (Universidade Federal do Rio de Janeiro) for the concession of the material, André Bello Bordeaux Rego for the Russian translations, to Dr. Graham Bird for his crucial suggestions for this work and two anonymous reviewers which provided helpful comments and improved the quality of this paper and Dr. Thiago Silva-Soares for the suggestion of the name T. brevicorpus. We also thank CNPq (Conselho Nacional de Desenvolvimento Científico e Tecnológico) for the concession of M.Sc. scholarships to JL. The junior author would like to thank also to CAPES (Coordenaçáo de Aperfeiçoamento de Pessoal de Nível Superior) for the grant (Scholarship Grant number: 5428/10-6).

\section{REFERENCES}

Anderson, G. 2013. Tanaidacea Classification, February 21, 2013. Available at http://peracarida.usm.edu/ TanaidaceaTaxa.pdf. Accessed on 27 November 2014.

Bird, G.J. and Bamber, R.N. 2000. Additions to the tanaidomorph Tanaidacea (Crustacea: Peracarida) of Hong Kong. p. 65-104. In: B. Morton (ed), The Marine Flora and Fauna of Hong Kong and Southern China V. Proceedings of the Tenth International Marine Biological Workshop: The Marine Flora and Fauna of Hong Kong and Southern China. Hong Kong, 6-26 April, 1998. Hong Kong, Hong Kong University Press.
Bird, G.J. and Larsen, K. 2009. Tanaidacean phylogeny - the second step: the basal paratanaoidean families (Crustacea: Malacostraca). Arthropod Systematics and Phylogeny, 67(2): 137-158.

Błażewicz-Paszkowycz, M. and Bamber, R.N. 2012. The Shallow-water Tanaidacea (Arthropoda: Malacostraca: Peracarida) of the Bass Strait, Victoria, Australia (other than the Tanaidae). Memoirs of Museum Victoria, 69: 1-235.

Lang, K. 1949. Contribution to the systematics and synonymics of the Tanaidacea. Arkiv for Zoologie, Series 1, 42(18): 1-14.

Lang, K. 1967. Taxonomische und phylogenetische Untersuchungen über die Tanaidaceen. 3. Der Umfang der Familien Tanaidae Sars, Lang und Paratanaidae Lang nebst Bermerkungen über den taxonomischen Wert der Mandibeln und Maxillulae. Dazu eine taxonomischmonographische Darstellung der Gattung Tanaopis Sars. Arkiv för Zoologi, Series 2, 19: 343-368.

Larsen, K. 2003. Proposed new standardized anatomical terminology for the Tanaidacea (Peracarida). Journal of Crustacean Biology, 23(3): 644-661.

Larsen, K. and Wilson, G.D.F. 2002. Tanaidacean phylogeny, the first step: the superfamily Paratanaidoidea. Journal of Zoological Systematics and Evolutionary Research, 40(4): 205-222.

Sars, G.O. 1899. Isopoda. An Account of the Crustacea of Norway, 2: 1-270 (plates 1-48).

Sieg, J. 1976. Zum natürlichen System der Dikonophora Lang (Crustacea, Tanaidacea). Zeitschrift für Zoologischer Systematik und Evolutionsforschung, 14: 177-198.

Sieg, J. and Dojiri, M. 1991. Two new species and a new genus of the suborder Tanaidomorpha (Crustacea: Tanaidacea) from Californian waters. Journal of Natural History, 25: 1493-1512. 
